\section{Pulmonary dirofilariasis in a Caucasian patient with metastasised osteosarcoma in a non-endemic European region}

A 22-year-old Caucasian man with a history of metastatic chondroblastic osteosarcoma was wedge resected via open thoracotomy after surveillance follow-up in 2009 revealed suspicious multifocal bipulmonary lesions on a CT scan (figure 1). Five years previously, neoadjuvant and adjuvant chemotherapy

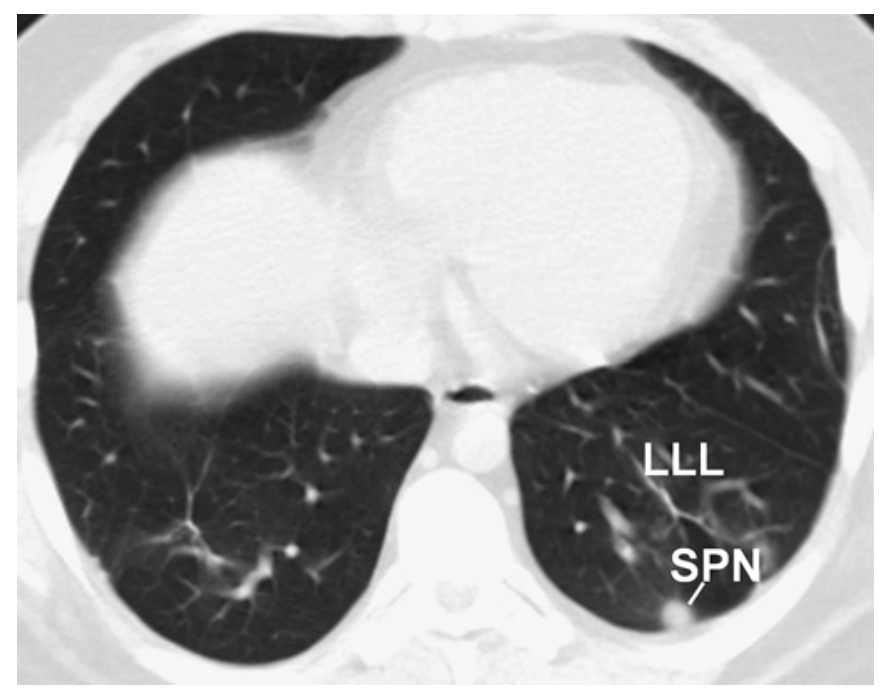

Figure 1 CT scan in lung window showing a solid pulmonary node (SPN) surrounded by scar tissue in the left lower lobe (LLL). This lesion presumably contained the nematode Dirofilaria repens.

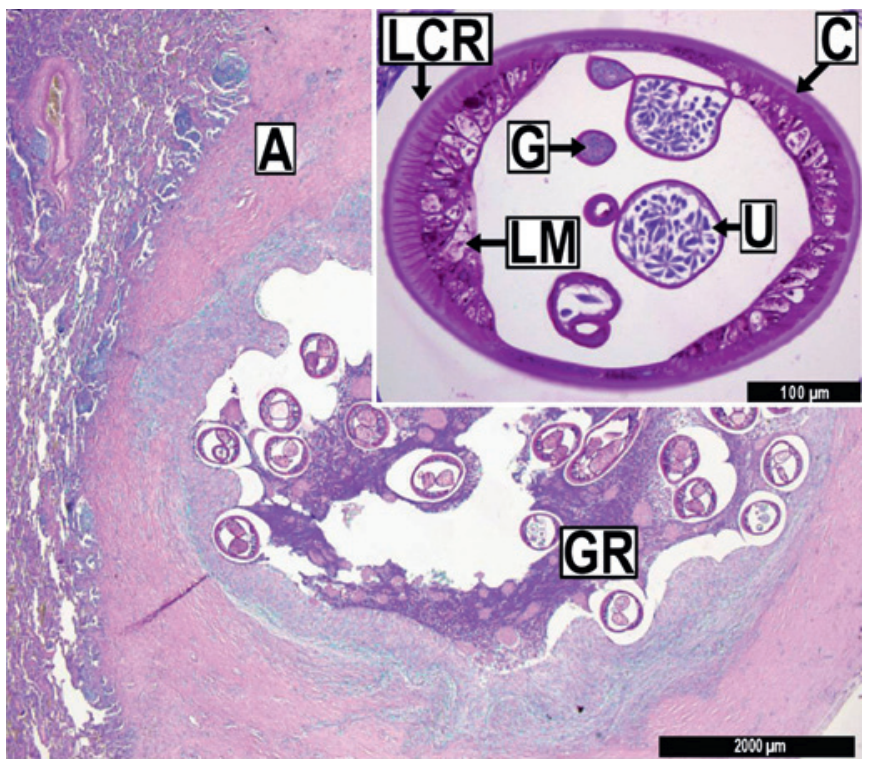

Figure 2 Periodic acid-Schiff-stained tissue sample of an embolised artery (A) containing sections of a female Dirofilaria repens (U, uterus) with its multilayered cuticle $(C)$ and external longitudinal cuticular ridges (LCR), longitudinal muscles (LM) and gut (G). The nematode is surrounded by a granulomatous reaction $(\mathrm{GR})$. Magnification $\times 10$; inlay $\times 40$.

\section{Learning points}

- Vector-borne transmissions of Dirofilaria repens occur in humans.

- Extended periods of immunosuppression can facilitate pulmonary dirofilariasis.

- Pulmonary dirofilariasis is a rare differential diagnosis of tumour-like lesions.

according to the COSS 96 protocol $^{1}$ had been given. Surveillance follow-up in 2009 showed new multifocal partly calcified pulmonary lesions in both lower lobes. Histologically, the resected lesion in the lower right lobe was vital tumour metastasis whereas the lesion in the lower left lobe showed a nonmalignant nodular granulomatous reaction to Dirofilaria repens (figure 2)

Dirofilariasis is a common vector-borne zoonosis. Occasionally, nematodes are transmitted by mosquitoes to subcutaneous tissues of humans. ${ }^{2}$ In extremely rare cases such as extended periods of immunosuppression, worms migrate to the lungs and cause asymptomatic granulomatous coin lesions. ${ }^{2}, 3$ Microscopically, typical pulmonary nodules display a central thrombosed artery and the parasite in various stages of degeneration, often surrounded by eosinophilia. ${ }^{4}$ To our knowledge, this is the first report of possible indigenous pulmonary dirofilariasis accompanying and mimicking lung metastasis in a patient with osteosarcoma.

\section{H Schmidt, ${ }^{1}$ U Dirksen, ${ }^{2}$ I Reiter-0wona, ${ }^{3}$ C Khurana, ${ }^{4}$ K Wiebe, ${ }^{5}$ R Wiewrodt, ${ }^{1}$ T Spieker ${ }^{6}$}

${ }^{1}$ Department of Medicine A, Pulmonary Division, University Hospital Münster, Münster, Germany; ${ }^{2}$ Department of Pediatric Hematology and Oncology, University Children's Hospital Münster, Münster, Germany: ${ }^{3}$ Institute of Medical Microbiology, Immunology and Parasitology, University Clinic Bonn, Bonn, Germany: ${ }^{4}$ Pediatric Oncology, Evangelisches Hospital Bielefeld, Bielefeld, Germany; ${ }^{5}$ Chest, Heart, and Vascular Surgery, University Hospital Münster, Münster, Germany; ${ }^{6}$ Gerhard-Domagk Institute of Pathology, University Hospital Münster, Münster, Germany

Correspondence to Lars Henning Schmidt, Department of Medicine A, Pulmonary Division, University Hospital Münster, 48149 Münster, Germany;

larshenning.schmidt@ukmuenster.de

RW and TS share the senior authorship of this manuscript.

Acknowledgements The authors thank Dr Sven Poppert, Bernhard Nocht Institute for Tropical Medicine, Hamburg, Germany for confirming the diagnosis of Dirofilaria repens by molecular biological methods

Competing interests None.

Patient consent Obtained.

Provenance and peer review Not commissioned; externally peer reviewed.

Accepted 23 October 2010

Published Online First 15 November 2010

Thorax 2011:66:270. doi:10.1136/thx.2010.142323

\section{REFERENCES}

Bielack S, Jürgens $H$, Jundt $G$, et al. Osteosarcoma: the COSS experience. Cancer Treat Res 2010;152:289-308.

2. Pampiglione S, Rivasi F, Gustinelli A. Dirofilarial human cases in the old world attributed to Dirofilaria immitis: a critical analysis. Histopathology 2009:54:192-204

3. Hemmersbach-Miller M, Delmont J, Hours C, et al. Pseudo-tumor of the lung, a rare clinical presentation of dirofilariasis. Presse Med 2005;34:109-10.

4. Asimacopoulos PJ, Katras A, Christie B. Pulmonary dirofilariasis. The largest single-hospital experience. Chest 1992;102:851-5. 